\title{
ECONOMIC ASPECTS OF CERVICAL CANCER SCREENING
}

\author{
Marc A. Koopmanschap, ${ }^{*}$ Koos Th. N. Lubbe, Gerrit J. van Oortmarssen, \\ Heleen M. A. van Agt, Marjolein van Ballegooijen and J. Dik F. Habbema \\ Department of Public Health and Social Medicine, Erasmus University Rotterdam, 3000 DR Rotterdam, \\ The Netherlands
}

\begin{abstract}
The results of a cost-effectiveness analysis of cervical cancer screening in The Netherlands are reported, emphasizing the analysis of the costs of screening and consequent diagnosis and treatment. Many organized screening policies are evaluated, differing in age-range and interval between screens. The cost estimates are based on organization charts. file studies and tariffs. The costs of screening itself are by far the most important cost component. Screening increases the costs of diagnosis. Costs for primary treatment only rise for large screening policies. Screening causes savings in costs of terminal treatment, but these are small compared with the costs of screening.

The costs per life-year gained for the most efficient policies amount to DFL 24,000 for the policy with 7 invitations per woman in a lifetime and rise considerably in case of more than 10 invitations. Cervical cancer screening appears to be less cost-effective than breast cancer screening, but compared with other services the results are comparatively good.

Implementing one of the efficient organized screening policies and discouraging spontaneous screening beyond that schedule leads to considerable savings. Moreover, many organized policies which are not efficient are still superior to spontaneous screening.
\end{abstract}

Key words-economic evaluation, costs, cost-effectiveness, cervical cancer, mass screening

\section{INTRODUCTION}

In The Netherlands 300 women per year die from cervical cancer, i.e. $0.5 \%$ of total female mortality. Symptoms of cervical cancer only occur when the cancer is already invasive. The pre-invasive stages of cervical cancer last on average about 15 years and can be detected by taking a Pap-smear. Almost $100 \%$ of the women with early detected pre-invasive stages can be cured.

A considerable part of the pre-invasive stages will not become invasive but will disappear (regress) spontaneously, especially in younger ages. Neither the smear nor further diagnosis can discern regressive from progressive pre-invasive stages, so any screening policy inevitably causes diagnosis and treatment and costs for women who would never have developed invasive cervical cancer. Taking Pap-smears has proved to be effective from the medical point of view: it can reduce cervical cancer mortality [1].

Discussion remains about the best screening policy: At what ages should women be screened? What is the appropriate interval between successive screens? Should women be invited? Who must take the smears, specially trained women or general practitioners and gynaecologists? To answer these questions it is necessary to estimate both health effects and costs of all possible courses of action. Moreover, costs and health effects of screening for cervical cancer should be evaluated in relation to other health services, given limited resources.

*Address correpondence to: M. A. Koopmanschap, Department of Public Health and Social Medicine, Erasmus University Rotterdam. P.O. Box 1738,3000 DR Rotterdam. The Netherlands.
The study on which this article has been based aims at determining which policies of cervical cancer screening in The Netherlands generate the best results in terms of health effects and costs [2]. To this end a prospective cost-effective analysis has been carried out in which epidemiological, medical and economic aspects were studied. This article describes the economic part and focuses on the following items:

-the costs of screening itself for various policies;

- the influence of screening on costs of diagnosis and treatment:

- the cost-effectiveness of the most favourable policies, as compared with other health care services.

\section{MATERIALS AND METHODS}

\section{In general}

During the last decade in The Netherlands, as in many other countries, early detection of cervical cancer was achieved in two ways. A screening programme was in operation for all women aged 35-54, who were invited at 3-year intervals. At the same time gynaecologists and general practitioners have taken many. smears, especially from younger women, without fixed intervals. As a result many women were screened very often. The efficiency of this combination of organized and spontaneous screening has been questioned.

In order to make recommendations about agegroups and intervals it is necessary to estimate costs and health effects of strategies in which only "organized' screening is performed. This means that women are invited regularly. The screening is performed 
exclusively by trained female personnel. No further smears are taken.

To estimate the impact of 'organization', costs and effects were also calculated for cases in which only spontaneous screening was performed. Early detection was by general practitioners and gynaecologists only, according to the distribution and frequency of smears as observed in The Netherlands during 1985-1987.

With respect to organized screening, cost and health effects were calculated for about 100 policies. The number of invitations per woman varied between 3 and 25 , the interval between two invitations ranged from 15 to 2 years. The age-group invited varied between the ages of 20 and 75 . Results were compared with the estimated costs and effects for the (hypothetical) situation where no early detection takes place: the zero-option.

A model for cervical cancer and the impact of screening on morbidity and mortality was developed. The micro-simulation programme MISCAN was used to calculate screening results and the effects of screening $[3,4]$. First, the model was fitted to data from the three Dutch pilot regions where organized screening took place during 1976-1984. Model assumptions about sensitivity of the Pap-smear and regression and duration of the pre-invasive disease stages were derived which offered a satisfactory fit with data concerning screen detected pre-invasive and invasive cases, interval cancers and mortality.

Next, the model was used to predict the health effects and costs of the policies. Data for the assumptions concerning organization and costs were collected by numerous interviews and analysis of cost accounts. They were integrated into the model by combining the predicted numbers of screens and the impact on diagnosis and treatment with the relevant cost-estimates.

The most important health effect of cervical cancer screening is the reduction in mortality, expressed in the number of life-years gained compared with the zero-option. From the total set of policies those with a maximum of life-years gained for a given level of costs were identified as efficient policies. Figure 1 shows the incremental costs and the life-years gained for all simulated policies. The marked points indicate efficient policies. The upper-left quadrant of these points is empty with neither policies producing more life-years gained for the same costs nor policies saving more life-years less expensively.

In the rest of this paper results will only be presented for a limited number of efficient organized screening policies and one variant of spontaneous screening.

\section{Cost calculations}

All possible costs and savings that screening policies bring about were identified and measured, taking the concept of social costs as our point of departure. This included non-financial costs and costs which do not fall upon the medical sector, but excluded value added tax. The costs of screening itself were derived by estimating the quantities and costs of each input of the screening programme. This resulted in cost functions reflecting the influence of fixed and variable costs and economies of scale.

The cost of diagnosis and treatment have been approximated by the tariffs charged (1987) in The Netherlands. An analysis of the true resource costs was beyond the scope of this study.

For each policy MISCAN calculated for each year the number of women invited, the number of women

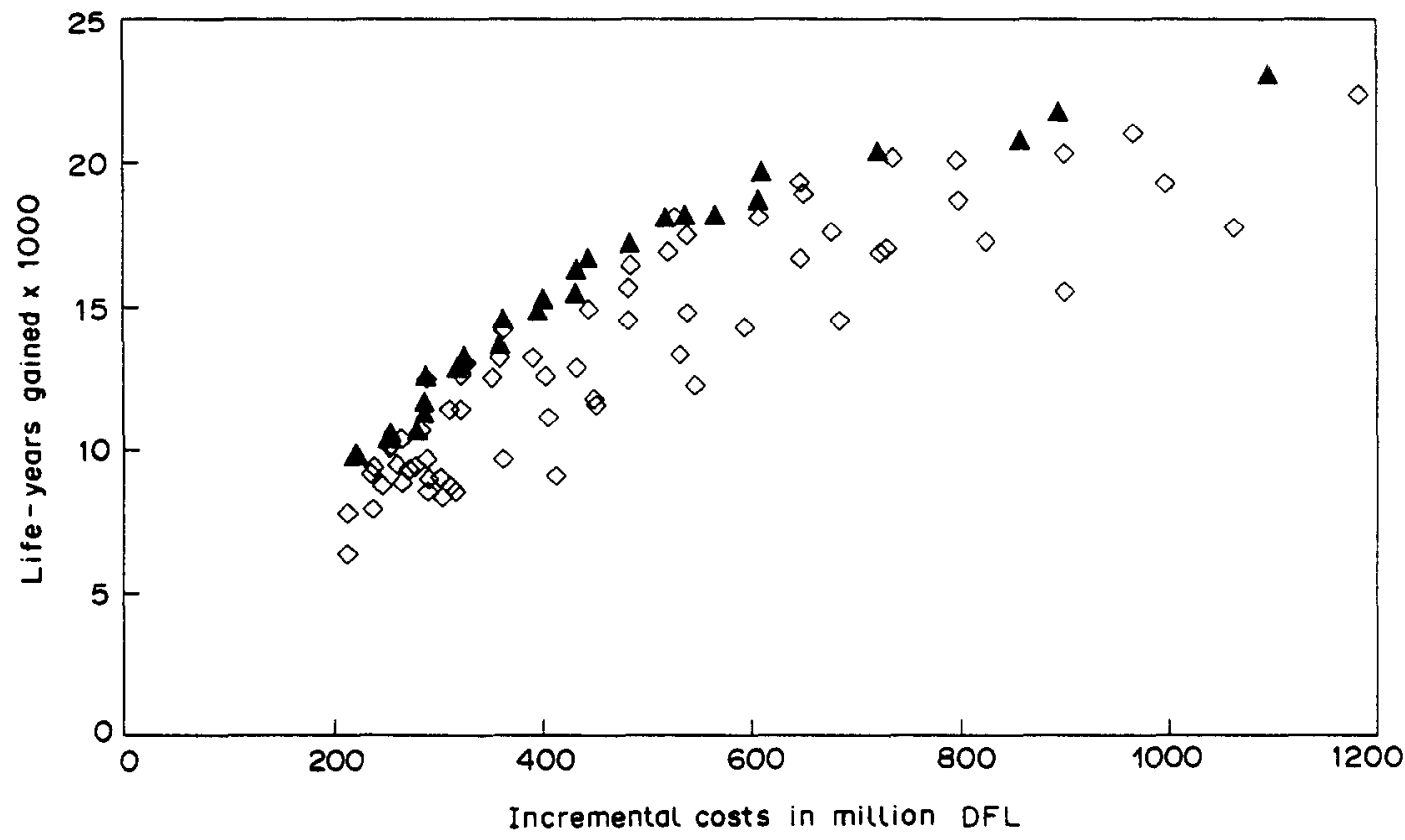

Fig. 1. Incremental social costs and life-years gained for about 100 different organized cervical cancer screening policies. Screening during 1988-2015. Discount rate $5 \%$. The marked points indicate the subset of efficient policies. 
Table 1. Financial costs of screening per year for organized screening policies with 250,000 and $1,000,000$ smears per year and spontaneous screening, in millions DFL (including $4 \%$ repedted smears, attendance rate $65 \%$ )

\begin{tabular}{lccc}
\hline & \multicolumn{2}{c}{ Organized policies } & Spontaneous \\
\cline { 2 - 2 } $\begin{array}{l}\text { Smears per } \\
\text { year }(\times 1000)\end{array}$ & $250(\%)$ & $1000(\%)$ & $910(\%)$ \\
\hline $\begin{array}{l}\text { Coordination } \\
\text { Invitations }\end{array}$ & $3.4(24)$ & $4.3(10)$ & - \\
$\quad$ and results & $1.1(8)$ & $4.4(10)$ & $1.2(3)$ \\
$\begin{array}{l}\text { Smear taking } \\
\text { Cytological }\end{array}$ & $3.5(24)$ & $13.3(31)$ & $14.3(40)$ \\
$\quad$ evaluation & $5.6(39)$ & $19.2(45)$ & $18.4(52)$ \\
Registration & $0.8(6)$ & $1.5(4)$ & $1.7(5)$ \\
$\begin{array}{l}\text { Total costs } \\
\text { of screening }\end{array}$ & $14.4(100)$ & $42.7(100)$ & $35.6(100)$ \\
$\begin{array}{l}\text { Per smear } \\
\text { (in guilders) }\end{array}$ & 58 & 43 & 39 \\
\hline
\end{tabular}

screened and the number of women needing diagnosis and/or therapy in various degrees. These outcomes, combined with the cost functions and tariffs produced the annual costs. Only after a considerable period the health effects of screening on cervical cancer become visible, while the costs of screening are important from the start. To permit adequate evaluation, the screening programme was assumed to be in operation during 1988-2015. After the year 2015 , the terminated programme will still cause health effects and changes in costs of diagnosis and treatment; these were calculated until 2088. Costs and effects were discounted to present values for 1988 with a discount rate of $5 \%$, stipulated by the Dutch government [5] and several health economists [6]. The estimated annual financial costs for government and insurers, including value added tax, will also be presented.

A fraction of women screened had a suspicious smear, leading to diagnosis and, if necessary, treatment. Therefore three categories of costs were relevant: costs for screening, diagnosis and treatment.

\section{Costs of screening}

The organization of the cervical mass screening in the three Dutch pilot regions and expert opinions about future developments were used to draw the outline of an efficient nationwide organization. This outline is only a tool to assess all relevant costs. The activities were carried out at three levels: local, regional and national (Table 1 ).

Local level. Each municipality sent a first invitation to the women in turn, by means of the up to date population registry. The women were screened in their municipality by a team of three trained smeartakers. The yearly capacity per team ranged from 19,000 (mobile unit) to 20,000 (static centre) smears. For a static centre the material costs were relatively low; use of a mobile unit increased the cost of taking smears by at least $25 \%$. The number of static and mobile units depended on the population density and the number of women screened. The time and travel costs of the women attending were relevant social costs (see Table 2). The avcrage time for travelling and screening was estimated at $50 \mathrm{~min}$ and was valued by $25 \%$ of the average net labour income per labour hour per household, according to binding advices [7]. Travel costs were estimated on the basis of public transport tariffs.

Regional level. With respect to cancer prevention The Netherlands was divided into 9 regions, each with on average about 800,000 female inhabitants. One coordination centre per region managed the screening teams, took care of local publicity and informed municipalities about the invitations. The centre sent test results to all women participating and re-invited non-participants. In cooperation with the cytological laboratory, quality control of the smears and training of the smear-takers was carried out. The centre was staffed by a manager, a medical consultant and administrative personnel. The workload and the costs varied according to the size of the programme.

The cytological laboratory evaluated the smears and registered the test results in a local data-base connected to a national data-base. On average, full time analysts evaluated approx. 7200 smears annually. Per 50,000 smears one senior analyst, one pathologist and two administrative employees are needed. Economies of scale existed for the costs of supervising and administrative personnel and part of the material costs.

National level. One centre took care of the national coordination and public relations. Using regional reports the policy is monitored and adapted if necessary. These costs did not depend on programme size. The costs of registration consisted of costs for the national data-base, costs of training the laboratory personnel and costs of local computer facilities. The national data-base also registered results of other pathological examinations, so the costs were only partially included.

Spontaneous screening. This incurred no costs of coordination and invitation. To estimate the costs of smear-taking, we assumed that all smears were taken

Table 2. Present value for 1988 of sucial custs of screening for three efficient policies and spontaneous screening during 1988-2015 in millions DFL. Discount rate 5\% (including 4\% repeat smears, attendance rate $65 \%$ ). Invitation schedule, number of invitations/age-range/interval

\begin{tabular}{lcccc}
\hline & & Efficient policies & & $\begin{array}{c}\text { Spontaneous } \\
\text { screening }\end{array}$ \\
\hline Invitation schedule & $7 / 37-73 / 6$ & $10 / 27-72 / 5$ & $16 / 26-74 / 3$ & - \\
Average number of smears $(\times 1000)$ & 390 & 610 & 975 & 910 \\
Coordination & 63 & 67 & 75 & -78 \\
Invitations and results & 23 & 35 & 56 & 18 \\
Smear taking & 77 & 118 & 187 & 270 \\
Cytological evaluation & 120 & 179 & 280 & 23 \\
Registration & 13 & 16 & 20 & 63 \\
Time and travel costs & 29 & 45 & 72 & 578 \\
Total costs of screening & 325 & 461 & 691 & \\
\hline
\end{tabular}


by general practitioners and calculated the labour costs of the general practitioner, the assistant and the material costs. Although this resulted in underestimating the costs since gynaecologists also took many smears which were more expensive, on the other hand, during a visit other medical activities may be performed as well, so only part of the costs should be attributed to the smear.

\section{Costs of diagnosis and treatment}

There are two situations where diagnosis and treatment take place: after an abnormal preventative smear or in case of possible symptoms of cervical cancer. In the zero-option, without any early detection, costs of diagnosis and treatment are only important for women with symptoms. In our study a detailed scheme was constructed to determine which diagnostic and therapeutic procedures took place for women having a suspicious smear. This scheme was subdivided by stage of disease and enabled us to calculate changes in number and types of procedures and costs when the stage-distribution of diagnosed (pre)-cancers alters due to screening. For more details see Ref. [8].

\section{RESULTS}

\section{Costs of screening}

In Table 1 the annual financial costs of screening are summarized for two different sizes of policies. Table 2 shows the present value of the social costs of policies, operational during 1988-2015, taking into account the demographic situation. The Results in Tables 1 and 2 include the costs of $4 \%$ repeat smears taken because of insufficient quality of the first smear.
The average attendance rate assumed is $65 \%$, as observed in the pilot regions. The total financial costs per smear range from DFL 58 for 250.000 annual smears to DFL 43 for $1,000,000$ smears per year. The economies of scale are considerable, chiefly because the costs for coordination and registration are almost fixed. The costs of coordination, registration and sending of invitations and test results form a substantial part of the total screening costs $(15-30 \%)$, whatever the size of the policy. The 'medical activities' smear-taking and cytological evaluation, make up $60-75 \%$ of the total costs. Time and travel costs account for $10 \%$ of the social costs of the screening (see Table 2).

The last column in Tables 1 and 2 indicates the costs of spontaneous screening on a scale of approx. 910,000 smears per year resembling the situation in The Netherlands during 1985-1987. The costs per smear are relatively low, the total costs of the spontaneous screening are considerable, due to the large number of smears.

\section{Costs of diagnosis and treatment}

The costs of diagnosis and treatment were computed by combining the relevant number of women with the schemes for diagnosis and treatment and the tariffs charged. Figure 2 depicts the extra costs and savings for diagnosis and treatment induced by two different organized screening policies, compared with the zero-option. The data are not discounted (Table 3 will show discounted costs).

Positive smears induce costs for diagnosis (DS). The more screening, the more positive test results, the higher the costs of diagnosis. Duc to screening the number of women with symptoms diminishes,

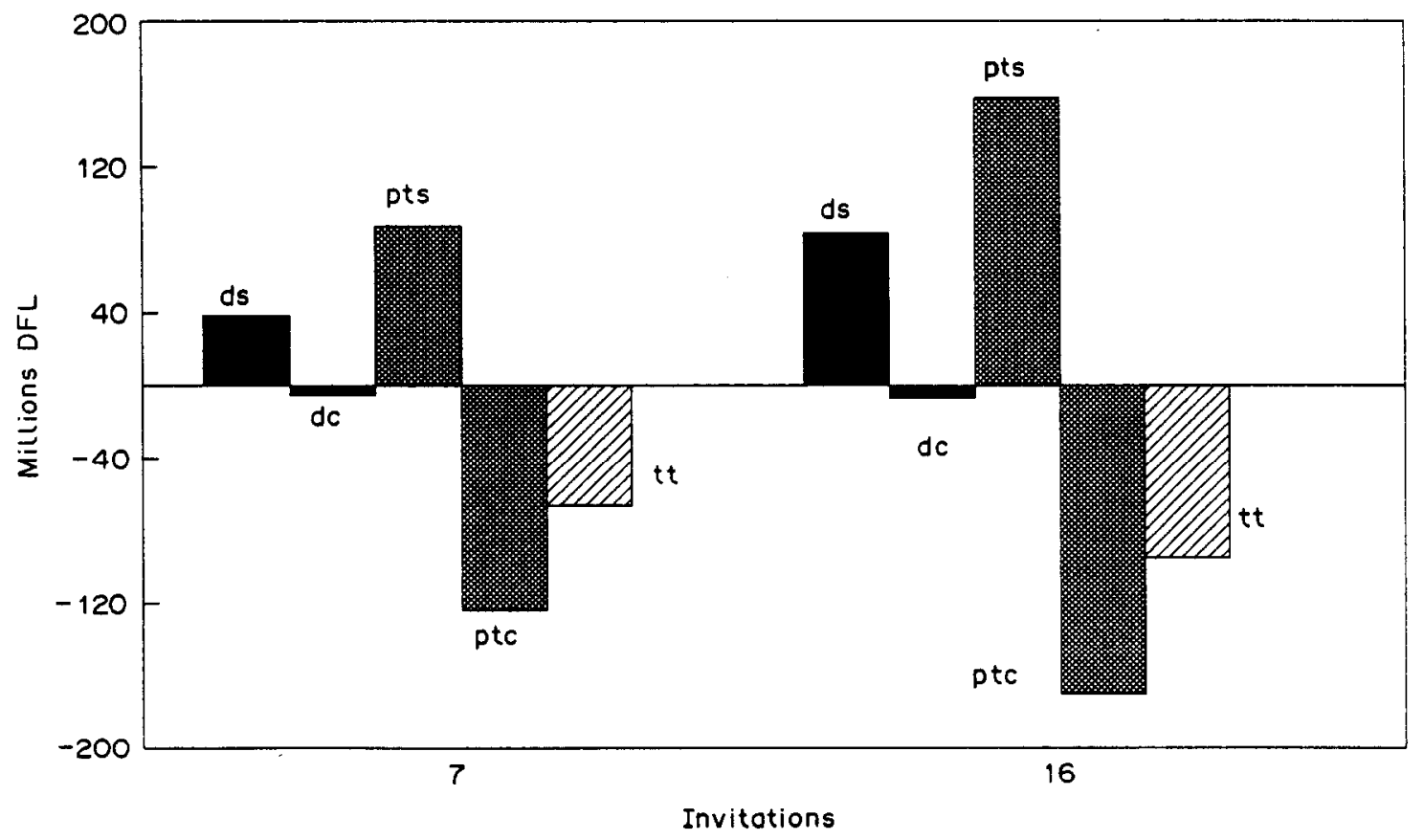

Fig. 2. Extra social costs and savings for diagnosis and treatment compared with no eariy detection, for two efficient policies, in millions DFL. No discounting. ds = extra costs of diagnosis induced by screening; $d c=$ savings in costs of diagnosis; pts = extra costs of primary treatment induced by screening: $\mathrm{ptc}=$ savings in costs of primary treatment; $\mathrm{tt}=$ savings in costs of terminal treatment 
Table 3. Present value for 1988 of social costs of screening, diagnosis, treatment and incremental costs for three efficient policies, spontaneous screening and no early detection in millions DFL. Period 1988-2088. Discount rate 5\%

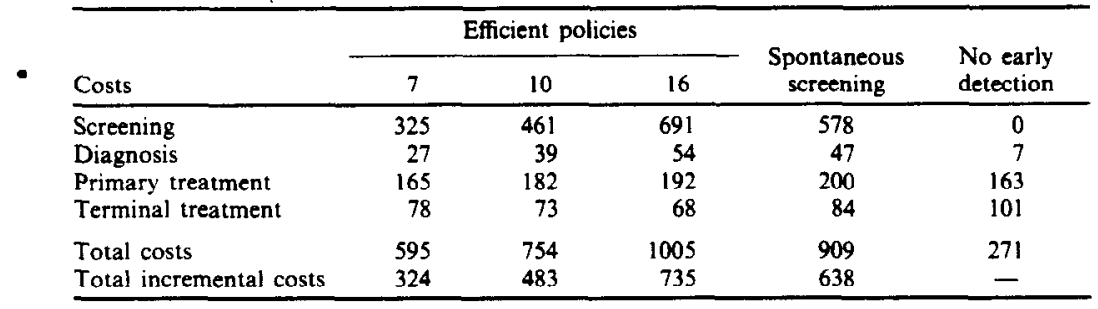

causing a small saving (DC). Screening increases the number of women with diagnosed precancers, which incurs costs for primary treatment (PTS). These costs consist of two parts: costs for women detected by screening, who would develop invasive cancer without screening and costs for women with regressive lesions which never would become invasive. The prevention of invasive cancers permits substantial savings on primary treatment (PTC). With more intensive screening savings (PTC) rise, but the increase of the extra costs (PTS) is even sharper, since the proportion of regressive lesions increases.

The reduction of invasive cancers causes a decrease in mortality. The savings on terminal treatment (TT) increase gradually with more intensive screening.

The savings induced by screening lag behind the extra costs. Discounting gives the results shown in Table 3. The discounted costs of diagnosis increases, particularly with more intensive screening. Screening with 7 invitations leaves the costs of primary treatment on balance unaltered. Intensifying screening means that the extra costs of primary treatment outweigh the savings at an increasing rate. Terminal treatment still produces savings, although discounting diminishes the absolute level. Spontaneous screening generates a high level of costs for primary treatment but this does not produce large savings on terminal treatment because the mortality reduction is relatively small.

\section{Total costs}

The costs of screening are by far the most important part of the total costs, especially for more intensive screening programmes. Costs of diagnosis and treatment are relatively small. Savings are to be expected on terminal treatment, but are rather modest. The total incremental costs compared with the zero-option range from 324 million DFL in the case of 7 invitations per woman to 735 million DFL if women are invited 16 times. The incremental costs of spontaneous screening exceeds 600 million DFL.

\section{Cost-effectiveness}

The cost-effectiveness of the policies is expressed as the incremental social costs per life-year gained. Table 4 shows the results for three efficient organized screening policies and one level of spontaneous screening.

The additional health effects of intensifying a screening policy diminish rapidly. An increase from 7 to 10 invitations per woman results in an additional number of 3900 life-years gained, as an increase from 10 to 16 invitations only saves 2900 life-years extra. The incremental costs rise faster, more or less proportional to the number of invitations. Consequently the incremental costs per life-year gained increases if screening is intensified. The efficient policy with 7 invitations per woman costs about 24,000 guilders per life-year gained. For the policy with 10 invitations the cost amounts to 28,000 guilders. For policies with more than 10 invitations the cost-effectiveness deteriorates rapidly.

Spontaneous screening on a scale reflecting recent Dutch practice generates a number of life-years gained equal to the efficient policy with 7 invitations, but the social costs are twice as high, When spontaneous screening takes place data indicate that part of the women, especially younger women, is screened very frequently (yearly), whereas the rest of the women only rarely has a screen and hence the health effects are limited. If no spontaneous screening is performed and the efficient policy with 7 invitations were to be implemented, 20 million guilders per year (financial costs) could be saved.

For a comparison of the efficient policies as well as for comparison with other health interventions, the marginal cost-effectiveness is important. Table 4 shows that the extra costs per extra life-year gained rise rapidly when switching from 7 to 10 invitations. Further intensifying the policy yields a considerable increase in marginal cost-effectiveness.

To ensure a rational health services budget, costeffectiveness should be compared with other services.

Table 4. Present values for 1988 of incremental social costs, life years gained and cost-effectiveness of three efficient screening policies and spontaneous screening. All outcomes are compared with the zero-option. Discount rate $5 \%$

\begin{tabular}{lcccc}
\hline & \multicolumn{3}{c}{ Efficient policies } & Spontaneous \\
\cline { 2 - 5 } & 7 & 10 & 16 & \begin{tabular}{c} 
screening \\
\hline Incremental social costs (millions DFL)
\end{tabular} \\
Life-years gained $(\times 1000)$ & 324 & 483 & 735 & 638 \\
Costs per life-year gained $(\times 1000$ DFL) & 13.3 & 17.2 & 20.1 & 13.4 \\
Marginal costs per life-year gained & 24.3 & 28 & 36.5 & 47.6 \\
$(\times 1000$ DFL) & 24.3 & 40.8 & 86.9 & - \\
\hline
\end{tabular}


Table 5 . Influence of the discount rate on social costs, health effects and cost-effectiveness for the efficient policy with 7 invitations. The percentages compared-with no discounting are in parentheses

\begin{tabular}{lccr}
\hline & \multicolumn{3}{c}{ Discount rate } \\
\cline { 2 - 4 } & $0 \%$ & $5 \%$ & $7 \%$ \\
\hline Incremental costs & 510 & 324 & 277 \\
(millions DFL) & $(100 \%)$ & $(64 \%)$ & $(54 \%)$ \\
Life-years gained & 68,300 & 13,300 & 7900 \\
& $(100 \%)$ & $(28 \%)$ & $(12 \%)$ \\
Costs per life-year gained & 7500 & 24,300 & 35,000 \\
(DFL) & $(100 \%)$ & $(324 \%)$ & $(467 \%)$ \\
\hline
\end{tabular}

A recent Dutch study of breast cancer screening using the same methodological assumptions [9] reported that breast cancer screening with 10 invitations per woman cost DFL 9700 per life-year gained. Further extension to 15 invitations cost DFL 25,800 per extra life-year gained. For the same number of invitations cervical cancer screening appears to be less attractive. Comparison with other health care services is difficult because of methodological differences between analyses. However, in Torrance's widely cited table of cost-effectiveness ratio's [10] the efficient policies of cervical cancer screening with 7 or 10 invitations have an intermediate cost-effectiveness ratio.

\section{Sensitivity analysis}

Cytological evaluation. On the basis of our recently collected data a workload of 7200 smears per analyst per year has been estimated. Assuming that the cytology is regionally concentrated so that expertise can attain a sufficient level, our preliminary view is that 7200 is a reasonable workload.

However, Dutch pathologists recently agreed [11] that if $85 \%$ of the smears are preventive $(15 \%$ of the smears are from women with symptoms) the optimal workload to aim for is 5000 smears. With $100 \%$ preventative smears the workload would then be about 5500 smears. The costs of cytological evaluation would increase $15 \%$, total costs of screening and the costs per life-year gained would rise $6 \%$, compared with the results presented. This workload forms the subject of a future study.

Discount rate. Results were also calculated using discount rates of 0 and $7 \%$. Table 5 shows that discounting at a rate of $7 \%$ implies that the number of discounted life-years gained is only $12 \%$ of the amount without discounting, whereas the incremental costs are still $54 \%$ of the amount without discounting. This confirms the importance of using a uniform discount rate in cost-effectiveness analyses.

\section{DISCUSSION}

\section{Quality of life}

Comparison of cervical cancer screening with other health care services should be based on costs per quality of life-year gained. Our study did not produce QALY-estimates, but two extreme variants were calculated to indicate the possible impact of quality adjustments. In the 'weak variant' the quality of life reductions for invasive cancer or being treated by hysterectomy or radiotherapy are assumed to be modest. In the 'strong variant' these reductions are greater and they are also applied to larger groups of women in less severe circumstances such as women with false-positive smears [2].

For efficient policy with 7 invitations the weak variant produces slightly more QALY's gained than without adjustment. The strong variant results in about $25 \%$ less QALY's. We expect that empirical research will produce estimates between these two extremes.

\section{Other studies}

Comparison with other studies of cervical cancer screening is difficult for three reasons. First, the number of serious cost-effectiveness analyses on this subject is very limited. Second, some of them deal with the epidemiology in detail but use only superficial economic assumptions [12]. Third, the only detailed study of economic aspects [13] focused on screening in a clinical setting which is different from a mass screening programme with respect to organization and costs.

\section{Organized and spontaneous screening}

In The Netherlands, as in many other countries, a combination of organized and spontaneous screening existed in the past and will probably continue in the future. The results of some of these combinations were calculated. They incur high costs for an intermediate level of health effects, because some women are screened in both contexts.

\section{Screening in the future}

In The Netherlands the organized screening programme terminated in 1984 was resumed in 1989. Women are invited from age 35 up to age 53 with a 3 -year interval, this in spite of our findings that such a schedule is not efficient (in efficient policies screening is performed up to 70 years). The costs per life-year gained amount to DFL 36,400 , assuming the organization described in this paper. However, in the new setting the general practitioners take the smears; the rest of the organization is not yet entirely established. Our preliminary research indicates that if the coordination of the activities of 6000 general practitioners and quality control is performed seriously the costs will not be much lower than reported here. The level of health effects is very uncertain, because the attendance rate and the quality of smears in the new setting remain unknown.

Our findings did not result immediately in any policy change. The government department of health care has maintained its old policy for organizational reasons. Further study of the effectiveness of screening in women older than age 55 will be carried out, which may influence the policy in the near future.

\section{CONCLUSIONS}

A large part of the costs of cervical cancer screening programmes consist of the costs of screening itself. Screening enlarges the costs of diagnosis. Costs of primary treatment remain unaltered for efficient policies with 10 or less invitations, but increase in case of more invitations. Costs of terminal treatment decline. 
The incremental costs per life-year gained are about 24,000 guilders for the efficient policy with 7 invitations and increases to 36,000 in case of 16 invitations per woman. The substantial influence of the discount rate on the cost-effectiveness confirms the need of uniform methodology.

Cervical cancer screening is less cost-effective than breast cancer screening on the same scale. Compared with other health care services the efficient policies with 7 or 10 invitations are cost-effective. For the same amount of cost, organized screening programmes are always superior to spontaneous screening. Discouraging spontaneous screening in The Netherlands and replacing it by efficient policy with 7 invitations per woman would save over 20 million guilders (financial costs) per year, without any rise in cervical cancer mortality. The Dutch public health insurance funds are trying to restrict spontaneous screening, except for situations in which women have possible cervical cancer-related symptoms, a small minority of the cases.

Acknowledgements-We thank Martin van Ineveld and Frans Rutten for their critical review of previous versions of this article. This study was financed by the Prevention Fund.

\section{REFERENCES}

1. Day N. E. Effect of cervical cancer screening in Scandinavia. Obstet. Gynec. 63, 714-718, 1984.

2. Habbema J. D. F., Lubbe J. Th. N., Agt H. M. E. van, Ballegooijen $M$. van, Koopmanschap $M$. A. and Oortmarssen G. J. van. Costs and Effects of Mass Screening on Cervical Cancer (in Dutch). Erasmus University, Rotterdam. 1988.
3. Habbema J. D. F., Oortmarssen G. J. van, Lubbe J. Th. N. and Maas P. J. van der. The MISCAN simulation program for the evaluation of screening for disease. Computer Meth. Prog. Biomed. 20, 79-83, 1984.

4. Habbema J. D. F., Lubbe J. Th. N., Oortmarssen G. J. van and Maas P. J. van der. A simulation approach to cost-effectiveness and cost-benefit calculations of screening for the early detection of disease. Eur. J. Opl Res. 29, 159-166, 1987.

5. Klaassen L. H. Studygroup discounting. In The Role of the Discount Rate in Policy-Making (in Dutch). Dutch Government, Department of Finance, The Hague, 1985.

6. Russell L. B. Evaluating Preventative Care. The Brookings Institution, Washington, DC, 1987.

7. Commission for development of analysis for policymaking (COBA). Second Report: Standards for Cost Benefit Analysis (in Dutch). Dutch Government. Department of Finance, The Hague, 1975.

8. Ballegooijen $M$. van. Diagnosis and treatment of cervical cancer (in Dutch). See Ref. [2], pp. B1-B52.

9. Maas P. J. van der el al. The cost-effectiveness of breast cancer screening. Int. J. Cancer 43, 1055-1060, 1989.

10. Torrance $G$. W. Measurement of health state utilities for economic appraisal. J. Hlth Econ. 5, 1-30, 1986.

11. Vooijs G. P. On behalf of the cytology commission of the Dutch pathologists association. Letter to the Dutch pathologists, 1987.

12. Parkin D. M. and Moss S. M. An evaluation of screening policies for cervical cancer in England and Wales using a simulation model. J. Epidem. Commun. Hlth 40, 143-153, 1986.

13. Luce B. R. The Implications of Cost Effectiveness Analysis of Medical Technology, An Application to Cervical Cancer Screening. Office for Technology Assessment (OTA) Congres, USA Washington, 1981. 